\title{
Cure of systemic arterial hypertension after hepatic adenoma laparoscopic resection: case report
}

\begin{abstract}
Background: Hepatic adenoma (HA) is relatively rare hepatic neoplasm. HA has been associated with use of oral contraceptives, hepatic deposit diseases or even abusive use of anabolic steroids. Generally, symptomatic uncomplicated HA presents abdominal pain, compression of loco regional structures or epigastric discomfort Gold-standard treatment has been surgical resection mainly by means laparoscopic approach.

Case report: Author reports one case of $\mathrm{HA}(4 \mathrm{~cm})$ in young female into segment $\mathrm{V}$ of the liver associated a high level of both renin and aldosterone besides systemic arterial hypertension. She was investigated for secondary arterial hypertension where was observed no positive findings. This patient wished to become pregnant, then she was submitted an AH enucleation by means laparoscopic approach. Subsequently, after $\mathrm{AH}$ resection, seric levels of both substances decreased for normal levels and systemic arterial hypertension was cured. Actually, two years after surgical resection, this patient presents normal level of both renin and aldosterone. Her arterial pression is under control without use of anti-hypertensive drugs.
\end{abstract}

Keywords: adenoma, hepatic neoplasms, liver, hepatectomy, renin
Volume 3 Issue 5 - 2017

\author{
Pais Costa SR \\ Department of Surgery, Brasilia Hospital, Brazil
}

Correspondence: Sergio Renato Pais Costa, $\mathrm{PhD}$ and MSC in Surgery from Federal University of São Paulo (UNIFESP), HPB Surgeon at Brasília Hospital, Federal District, SEPS 710/910, Conjunto D-Sala 330, Brazil, CEP 70390-108, Email srenatopaiscosta@hotmail.com

Received: September 30, 2017 | Published: October 31, 2017
Abbrevations: HA, hepatic adenoma; OC, oral contraceptives; AAS, anabolic androgenic steroids; HNF1- $\alpha$, hepatocyte nuclear factor-1- $\alpha$; IL6ST, il-6 signal transducer; SAH, systemic arterial hypertension; US, ultrasound; CT, computed tomography; MNR, magnetic nuclear resonance

\section{Introduction}

Hepatic adenoma (HA) presents as a relatively uncommon primary benign neoplasm of hepatocellular origin that attains more frequently young women. Generally, HA has been associated with use of oral contraceptives (OCs). Hepatic diseases as Glycogen storage disease has also been associated to development of the HA beyond other conditions such diabetes mellitus, pregnancy, Fanconi anemia, Hurler disease, Familial adenomatous polyposis and Tyrosinemia. ${ }^{1-7}$ Seldom, HA has been associated with abusive use of anabolic androgenic steroids manly (AAS) by bodybuilders. ${ }^{8}$ Generally, these tumors are detected by means incidental finding due image examinations techniques. ${ }^{9}$ When symptomatic, $\mathrm{AH}$ may present different symptoms as hepatomegaly, right upper quadrant discomfort, pain, compression of neighboring organs, or intraperitoneal hemorrhage. Intraoperative diagnosis is occasionally established only at surgical exploration due hemorrhagic shock. ${ }^{1-10}$ Tumor rupture may be observed in pregnant women and it is frequently associated with bulky tumors. This clinical picture may leave high mortality. ${ }^{1-10} \mathrm{HA}$ have also been associated a malign transformation. This association is also observed in large tumors. ${ }^{2,4}$ Nowadays, AH biologic behavior has been studied, mainly based in different prognosis that may be found based in diverse mutations. Three varieties of mutations are well established in literature. Therefore, $\mathrm{AH}$ is subdivided in accordance with its mutation, actually $\mathrm{AH}$ follows the following classification:

i. Steatotic HA caused by hepatocyte nuclear factor $1-\alpha(\mathrm{HNF} 1-\alpha)$ mutation.

ii. Inflammatory AH caused by IL-6 signal transducer (IL6ST) mutation.

\section{iii. $\mathrm{AH}$ associated with beta catenin mutation. ${ }^{10,11}$}

Choice treatment has been surgical resection by formal hepatectomy or enucleation. ${ }^{4,10,11}$ This is the preferential approach for the following circumstances: symptomatic patients, patients who wish to make pregnant, $\mathrm{AH}$ in men, $\mathrm{AH}$ with beta catenin mutation or even large tumors $>5 \mathrm{~cm} .{ }^{1-10}$ Actually, laparoscopic approach has become golden standard because carries low morbidity, fast recovery, and cosmetic advantages. ${ }^{2,6,7,12,13}$ The present author described a case of HA in a young woman without previous history of use either OC's or AAS. Suddenly, this patient began to present systemic arterial hypertension (SAH). Her SAH was associated a seric high levels of both renin and aldosterone. The cause of seric elevation of these substances was unknown; despite it had been hardly searched. It was observed none radiologic lesions except AH. This patient was submitted a laparoscopic enucleation of HA with success. So, after surgical resection was observed cure of systemic arterial hypertension.

\section{Case report}

A 32year-old female was referred to us for the appearance of incidental nodular lesion into segment $\mathrm{V}$ of the liver. This patient has ever used neither OC's nor AAS. She reported previous investigation of systemic arterial hypertension over last three months. She presented no familial history for SAH. Secondary arterial hypertension was exhaustively investigated, as renovascular hypertension as adrenal causes like pheochromocytoma and other lesions. All of them were excluded. Neuroendocrine tumors were also excluded since an Octreoscan was negative. Laboratory function tests were within normal limits. Hepatitis B surface, anti-HBs antibody and antihepatitis $\mathrm{C}$ virus antibody were negative. Serum tumor markers (CEA, CA 19, 9 and $\alpha$-fetoprotein) were also negative. She presented only seric high levels of both renin $(17,9 \mathrm{dl} / \mathrm{ml})$ and aldosterone $(16,0 \mathrm{dl} / \mathrm{ml})$. She used $1500 \mathrm{mg} /$ day of methyldopa because she wished to make pregnant. Abdominal computer tomography (CT) showed a hypervascular heterogeneous lesion measuring 40 X 38 X 36mm and localized in $\mathrm{V}$ hepatic segment very close from gallbladder 
(Figure 1). The lesion was well defined showing dyshomogenous density with irregular enhancement in the arterial phase and washout in late phase. CT findings suggested hepatic adenoma. Surgical resection was indicated due risk of rupture in pregnancy. The patient was brought to the laparoscopic resection that was performed without intercurrences. A $4 \mathrm{~cm}$ diameter of heterogeneous hemorrhagic hepatic lesion was observed in $\mathrm{V}$ hepatic segment (Figure 2). This lesion was enucleated and taken off by means endobag. The mass appeared as well circumscribed margins lesion with smooth external surface. The lesion was green-brown colored tumor with hemorrhagic areas. Microscopic examination showed the presence of mature, vacuolated hepatocytes; no portal or biliary structures were present, which confirmed inflammatory HA. Lesion presented hemorrhagic focus, but presented no malignant signals. Immuno-histochemistry evaluation showed IL6ST mutation which confirmed inflammatory AH. The patient stayed two days in Hospital. She recovered well without postoperative complications. Following surgery, as seric levels of both renin and aldosterone as arterial pression were decreased. After three months of surgical resection, they presented normal levels and SAH was resolved without use of anti-hypertensive drugs. At last follow-up examination at two years did not reveal the presence of any further focal lesions, and she presents well without SAH. The seric levels of both renin and aldosterone presented normal at last followup. This patient has used none anti-hypertensive drug.

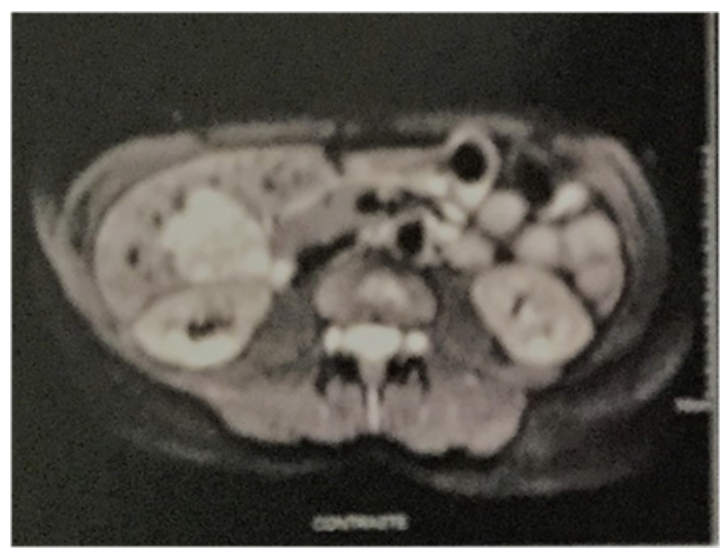

Figure I Magnetic Nuclear Resonance with gadolinium-Hypervascular heterogeneous lesion localized in $\mathrm{V}$ hepatic segment.

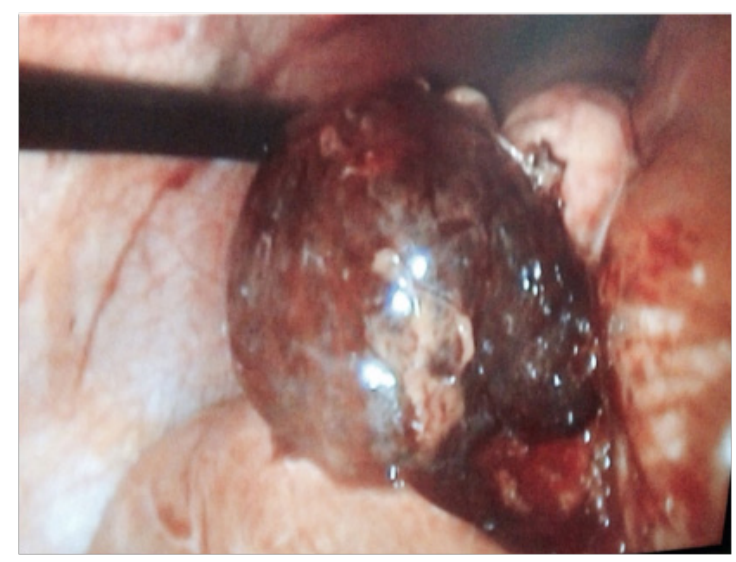

Figure 2 Laparoscopic resection of $\mathrm{AH}$.

\section{Discussion}

HA is a relatively rare hepatic benign lesion which is characterized by proliferation of hepatocytes. HA has been more frequently observed in young female (4:1) between 20 and 40years old. The pathogenesis seems unclear, nevertheless has been associated with use of the both OCs and AAS. ${ }^{1-5}$ More often than not, patients with HA have no symptoms therefore are incidentally diagnosed by radiologic methods as observed in this present case. For symptomatic cases, the initial presentation may be widely diverse. Abdominal pain, mass effect, epigastric fullness, or even hemorrhagic shock due tumor rupture may be seen on many symptomatic cases. ${ }^{1-7}$ Occasionally, large AH may lead to spontaneous rupture or haemorrhage and, in certain cases, even death. The chance of rupture is no negligible (15a $33 \%$ ), may be associated with high mortality, manly when performed emergency surgery (5a 10\%). Besides rupture, AH has been presented other major complication that is malignant transformation. Malignant transformation has varied too much, however it is estimated that 4a $11 \%$ of $\mathrm{AH}^{2-10}$ This complication is more frequent in male with Beta catenin mutation. To date, under author's knowledge, it was no reported association between $\mathrm{AH}$ and high seric levels of both renin and aldosterone leading SAH. In fact, nevertheless in literature review was no observed this association, the true cause effect is unknown in our view point. As neuroendocrine tumors as renovascular disease were excluded in this particular picture, therefore it leads to believe in a different biologic behaviour of this rare hepatic neoplasm. Radiologic diagnosis is the most important for AH detection. Ultrasound (US), Computed Tomography (CT) and magnetic nuclear resonance (MNR) of the abdomen are usually important for diagnosis. HA is a vascular tumor with predominant arterial supply in the classic cases, the findings are well vascularized and well defined solid lesion that is predominant in arterial phase on CT or MNR scan. ${ }^{9}$ Sometimes the lesion is dyshomogeneous as present case, mainly if haemorrhage is present. ${ }^{1,5,6}$ One of the problems that HA present is differential diagnosis with hepatocellular carcinoma (HCC) or even vascularized metastasis mainly neuroendocrine lesions. In fact, radiological findings in patient with HA are often similar to those in patients with HCC. In those cases, in which clinical, radiological, and histological distinctions between HA and HCC are difficult to determine, surgical resection, whenever possible has been advised. ${ }^{1-7}$ Conservative management may be generally used in small HA or even those steatotic (HNF1- $\alpha$ ) AHs, mainly those relational with use of the OCs or AAS. ${ }^{10,11}$ For AAS induced HA to stop the use of the AAS may sometimes to reduce the lesion. Rigid Follow-up by means of radiologic examinations (US or TC) every 6 months is absolutely necessary. ${ }^{10}$

When $\mathrm{AH}$ is inflammatory, large $(>5 \mathrm{~cm})$ or even it presents Beta catenin mutation surgical treatment has been advised by many authors. In these situations, as rupture as malignant transformation has been observed in literature. ${ }^{1-10}$ Also, woman who wishes to make pregnant must have surgical indication since tumor rupture during pregnancy is no negligible. This option also depends on case-by-case, in the present case where the lesion was superficial into segment $\mathrm{V}$ of the liver a tumor rupture has been described in literature. This emergency situation is very dangerous due hemoperitoneum and hypovolemic shock. In this case, tumor enucleation is enough and it presents low morbidity like observed by others. Laparoscopic hepatectomy has been a good option to the benign tumors such HA., ${ }^{72}$ The present author has reported this approach with great safety even in posterior lesions at right lobe. ${ }^{13}$ The present author has also used whenever possible sparing liver parenchyma resections mainly for benign tumors like HA. ${ }^{12,13} \mathrm{AH}$ behaviour has also depended of its molecular signature mainly based of mutation type. Therefore, for steatotic $\mathrm{AH}$ with HNF1- $\alpha$ mutation, conservative approach has been the main conduct because as rupture as malignant transformation has been very rare condition. Instead, when IL6ST mutation has been observed the 
risk of rupture is relatively more common like observed in present case besides AH malignant transformation. , $, 6,10,11$ The rupture of $\mathrm{AH}$ has been associated a high mortality principally for subcapsular tumors as present case and it is no rare during pregnancy. ${ }^{2,4}$ Finally, when Beta-catenin mutation has been observed a high risk of malignant transformation occurs mainly in men, where its resection seems to be main approach. . $67,7,10,11$ When HA is well managed by enucleation, the overall prognosis is good like observed in this case. Recurrence may occur in inflammatory $\mathrm{AH}$ and these patients should be followed. Even to the operated patients the follow-up should be done in regular medical visit and radiologic examinations..$^{1-7}$ At present case, a specific follow-up has been used to screen the seric levels of both renin and aldosterone besides radiologic examinations. However, to date two years after resection they are normal and her arterial pression presents under control without use of anti-hypertensive. In sum, though the real cause effect relation between $\mathrm{AH}$ and $\mathrm{SAH}$ were unknown in our view point, a resolution of SAH was observed in this case after $\mathrm{AH}$ resection. An objective finding as normalization of both renin and aldosterone seric levels after $\mathrm{AH}$ resection was observed in this case. It may lead to believe in association of $\mathrm{AH}$ and high levels of reninaldosterone with SAH.

\section{Acknowledgements}

None.

\section{Conflicts of interest}

Author declares that there is no conflict of interest.

\section{References}

1. Choi BY, Nguyen MH. The Diagnosis and Management of Benign Hepatic Tumors. J Clin Gastroenterol. 2005;39(5):401-412.

2. Laurent A, Dokmak S, Nault JC, et al. Europen experience of 573 liver resections for hepatocellular adenoma: cross-sectional study by AFCHCA-2013 study group. HPB (Oxford). 2016;18(9):748-755.
3. Herman P, Pugliese V, Machado MAC, et al. Hepatic Adenoma and Focal Nodular Hyperplasia: Differential Diagnosis and Treatment. World J Surg. 2000;24(5):372-376.

4. Ribeiro Junior MAF, Chaib E, Saad WA, et al. Surgical management of spontaneous ruptured hepatocellular adenoma. Clinics (Sao Paulo). 2009;64(8):775-779.

5. Szor DJ, Ursoline M, Herman P. Hepatic Adenoma. Arq Bras Cir Dig. 2013;26(3):219-222.

6. Bieze M, Busch ORC, Tanis PJ, et al. Outcomes of liver resection in hepatocellular adenoma and focal nodular hyperplasia. HPB (Oxford). 2014;16(2):140-149.

7. De'Angelis N, Memeo R, Calderaro J, et al. Open and laparoscopic resection of hepatocellular adenoma: trends over 23years at a specialist hepatobiliary unit. HPB (Oxford). 2014;16(9):783-788.

8. Pais-Costa SR, Lima OAT, Soares AF. Giant hepatic adenoma associated with anabolic-androgenic steroid abuse: case report. Arq Bras Cir Dig. 2012;25(3):180-182.

9. Grazioli L, Federle MP, Brancatelli G, et al. Hepatic adenomas: imaging and pathologic findings. Radiographics. 2001;21(4):877-892.

10. Liu JB, Baker MS. Benign Liver Tumors. In: Zyromski NJ, editor. Handbook of Hepato-Pancreato-Biliary Surgery. 1st ed. Philadelphia, USA: Wolters Kluwer; 2015.

11. Parks RW, Garden OJ, Gigot JF. Benign Liver Tumors. In: Garden OJ, Parks RW, editors. Hepatobiliary and Pancreatic Surgery. 5th ed. Philadelphia: Saunders \& Elsevier; 2014.

12. Pais-Costa SR, Araújo SMA, Lima AOT, et al. Laparoscopic Hepatectomy: indications and results from 18 resectable cases. Eisntein. 2011;9(3):343-349.

13. Costa SRP, Araujo SLM, Lima AOT, et al. Laparoscopic Right Posterior Sectioniectomy for Treating Hepatic Tumors. Arq Bras Cir Dig. 2010;23(4):275-279. 